\title{
Meta
}

Journal des traducteurs

Translators' Journal

\section{APTER, Ronnie et HERMAN, Mark (2016) : Translating for Singing. The Theory, Art and Craft of Translating Lyrics. Londres/New York: Bloomsbury, 282 p.}

\section{Marie Nadia Karsky}

Volume 62, numéro 2, août 2017

URI : https://id.erudit.org/iderudit/1041036ar

DOI : https://doi.org/10.7202/1041036ar

Aller au sommaire du numéro

Éditeur(s)

Les Presses de l’Université de Montréal

ISSN

0026-0452 (imprimé)

1492-1421 (numérique)

Découvrir la revue

Citer ce compte rendu

Karsky, M. N. (2017). Compte rendu de [APTER, Ronnie et HERMAN, Mark

(2016) : Translating for Singing. The Theory, Art and Craft of Translating Lyrics.

Londres/New York : Bloomsbury, 282 p.] Meta, 62(2), 469-471.

https://doi.org/10.7202/1041036ar d'utilisation que vous pouvez consulter en ligne. 
linguistique» (p. 121) chez Paiement, au «bilinguisme soustractif» (p. 129) de Dalpé, à «la "notion périmée de la langue" babélisée et théâtralisée» (p. 136) chez Leroux et «aux clignotements angloontariens et montréalais» (p. 170).

Si le théâtre franco-ontarien s'est fondé sur l'œuvre de Paiement et, en quelque sorte et de différentes manières la prolonge, le théâtre acadien s'est trouvé à un certain point dans l'ombre de $L a$ Sagouine. C'est au tour des dramaturges tels Rino Morin Rossignol, Herménégilde Chiasson et, plus récemment, Paul Bossé, Christian Essiambre et Philippe de Soldevila de pousser plus loin «le jeu théâtral au cœur du grouillement linguistique acadien» (p. 179). Comme le précise Chiasson, " on n'arrive pas à se brancher sur un langage, sur une particularité, et ça, c'est dur. Pour rendre compte de l'Acadie, il faudrait écrire en français, en chiac, en anglais, bref, il faudrait écrire sur toute une aliénation» (p. 188). Si Antonine Maillet avec La Sagouine et les textes consécutifs, et Laval Goupil, avec Tête d'eau, ont su «fixer une langue orale» (p. 182), elle ne représente, comme le démontre Nolette, qu'une partie de cette parole multiple qu'est l'Acadie. De Rossignol qui «invite tous les stéréotypes des régionalismes acadiens dans Le pique-nique (p. 187), à Chiasson chez qui les dialectes des personnages transmettent sur scène leur attachement à l'Acadie ainsi que leur engagement social et politique (p. 189), à une dramatisation du texte de France Daigle qui s'intitule Moncton-Sable, à 2001: A Space Odyssey, une pseudo-traduction signée Bossé, jusqu'aux Trois exils de Christian E (exil linguistique, exil géographique, exil théâtral) d'Essiambre et de Soldevila, le "grouillement linguistique» acadien se transforme en jeu théâtral. La circulation de cette dernière pièce qui a connu un grand succès d'abord à Ottawa et par la suite dans d'autres théâtres franco-canadiens, y compris ceux au Québec, les collaborations avec d'autres compagnies comme le TTF, ainsi que les traductions de Glen Nichols, y compris Traces, la traduction d'Empreintes, mettent en évidence l'intérêt de ce théâtre audelà de l'image folklorique de La Sagouine et «la construction de ces communautés hybrides sans frontières par le jeu et par l'humour» (p. 244).

Comme Nolette le précise dans sa conclusion, cette étude de la place de l'hétérolinguisme ou de l'inscription de la variabilité linguistique et de la traduction ludique dans le théâtre franco-canadien à l'extérieur du Québec invite les comparaisons entre la traduction ludique comme stratégie créatrice entre les trois zones, de l'ouest, de l'Ontario et de l'Acadie. Mais elle incite surtout à poser de nouveaux regards sur, par exemple, la division traditionnelle entre le spectacle vivant et la littérarité du texte ou, d'un aspect plutôt théorique, sur la traduction comme activité entre deux langues et deux cultures (p. 251). Il s'agit plutôt, et souvent, d'un jeu à l'intérieur d'une même langue hybride. Nolette nous propose donc non seulement une analyse fascinante et compréhensive des théâtres à la fois semblables et distincts, mais un élargissement des théories de la traduction, car selon elle, «la traduction ludique, c'est un véritable dialogue entre le domaine de la traductologie et celui des littératures franco-canadiennes, un dialogue qui les renouvelle tous les deux» (p. 251). Stimulant modèle que celui de Nolette.

Jane Koustas

Brock University, St. Catharines, Ontario

\section{RÉFÉRENCES}

Grutman, Rainier (2005): La textualisation de la diglossie dans les littératures francophones, dans Jean Morency, Hélène Destrempes, Denise Merkle et Martin Pâquet (dir.). Des cultures en contact: visions de l'Amérique $d u$ Nord francophone, Québec, Nota bene, 201223.

PAré, François (2003): La distance habitée. Hearst: Le Nordir.

Apter, Ronnie et Herman, Mark (2016): Translating for Singing. The Theory, Art and Craft of Translating Lyrics. Londres/New York: Bloomsbury, $282 \mathrm{p}$.

Dans Translating for Singing, Ronnie Apter et Mark Herman, tous deux traducteurs vers l'anglais de livrets d'opéra et plus généralement de textes chantés, présentent la nature, les difficultés et les joies de leur travail, tout en le théorisant et en historicisant la tâche du traducteur de ce genre de textes. Si l'ouvrage se borne à la traduction vers l'anglais, nul doute que les considérations des auteurs ne puissent servir à la réflexion sur la traduction d'opéras et de chants en d'autres langues. L'ouvrage se compose de 12 chapitres et d'une conclusion, et il est doté d'un index ainsi que d'une bibliographie très fournie. Des extraits d'airs traduits souvent donnés avec leur partition viennent amplement illustrer le propos, ce qui permet aux lecteurs le désirant de vérifier par eux-mêmes ce qui est avancé.

Le texte d'Apter et Herman est précédé d'une préface de Jonas Forssell, traducteur suédois, qui expose tout d'abord une contradiction fréquente dans l'opéra: genre scénique, voué à l'expression, ses œuvres sont souvent chantées dans des langues que la plupart des publics ne comprennent pas. Forssell soulève plusieurs questions qui incitent le lecteur à chercher des réponses dans les chapitres 
de l'ouvrage: comment dépasser la hiérarchisation des genres, en grande partie fondée sur des préjugés linguistiques et culturels, entre l'opéra, surtout lorsqu'il est chanté dans la «langue noble» qu'est l'italien, et les spectacles musicaux (comme la comédie musicale) souvent écrits en anglais? Comment, par conséquent, donner un statut à l'opéra en langue anglaise? L'avènement du surtitrage permet-il vraiment de dépasser la barrière de la langue, étant donné la concision de l'information communiquée? Forssell en appelle plutôt à traduire les opéras en anglais, lingua franca de la mondialisation, qu'il nomme cependant "vernacular». Là réside un des points qui nous surprend: si la traduction d'opéras en anglais peut certes contribuer à leur compréhension dans plusieurs pays, au nom de quelle politique linguistique et culturelle chanterait-on Verdi ou Wagner en anglais en Suède ou dans un autre pays non anglophone, plutôt que de le chanter dans la langue du pays? On imagine que ce serait avant tout pour des questions de rentabilité auxquelles l'opéra, lui non plus, ne peut échapper... Par ailleurs, si la traduction vient remplacer les sous-titres, les spectateurs sontils vraiment assurés de comprendre les paroles chantées? Cela suppose, de la part des chanteurs, une articulation excellente, qui n'est pas toujours monnaie courante, et certaines notes, aiguës ou graves, ne favorisent pas la diction. Telles sont les questions que l'on se pose à la lecture de cette introduction très stimulante, qui nous rend encore plus désireux d'aborder l'ouvrage de Apter et Herman.

Le premier chapitre, intitulé «Translation and music », inscrit la traduction des livrets d'opéra dans la recherche en traductologie, faisant un point bibliographique utile, mais surtout pose la question de savoir ce qui constitue une bonne traduction vers l'anglais de paroles chantées (que ce soit pour l'opéra, le lied, la comédie musicale, l'oratorio...). Comment le texte traduit peut-il à la fois fonctionner en scène, être chantable, respecter les exigences de la musique, tout en constituant une traduction et non une adaptation ou une réécriture? Cette question sert de fil conducteur aux différents chapitres qui apportent progressivement des éléments de réponse plus précis. Les auteurs définissent la tâche du traducteur de livrets musicaux comme consistant à traduire les mots dans leur relation à la musique, rappelant que paroles et musique forment un tout, et revenant au passage sur la polémique "prima la musica dopo le parole» pour signifier sa vanité. Dans le deuxième chapitre, ils font un rappel utile de l'histoire de la traduction d'opéras, évoquant la suprématie historiquement accordée à l'italien et les changements qui se sont produits depuis les années 1950 avec l'internationalisation accrue des chanteurs. Dans les pays anglophones, certains théâtres continuent à faire chanter les œuvres dans des traductions anglaises, mais il est rare, en général, que les chanteurs disposent de livrets traduits autrement que dans des traductions littérales. Les notes et les paroles ne correspondent pas précisément et il est difficile pour les chanteurs anglophones de comprendre véritablement ce qu'ils chantent. Les auteurs dénombrent ensuite, dans ce chapitre puis au cours de l'ouvrage, les difficultés principales de traduction que posent les textes chantés: les plus évidentes sont liées à la différence de systèmes prosodiques; le traducteur de livrets musicaux se verra donc obligé de tenir compte des critères que sont le rythme, la rime, le sens, la correspondance entre syllabe et note, la fluidité syntaxique, ainsi que les caractéristiques des personnages (idiolectes, registres de langue employés...). Certaines modifications musicales minimales sont possibles, présentées par les auteurs dans un tableau.

Une fois ces principes posés, les auteurs confrontent, dans les cinq chapitres suivants (soit les chapitres 3 à 7), la traduction de textes pour le chant à différents critères traductologiques: la traduction sera-t-elle faite de manière sourcière ou cibliste? Quels sont les éléments d'adaptation qui entrent en jeu? Qu'est-ce qui justifie les retraductions pour l'opéra? Comment traiter des décalages historiques, géographiques, culturels, de tous les éléments qui passent par le filtre de la censure ou de l'autocensure, passées et présentes, et du politiquement correct? Les exemples donnés, de l'Eraclea de Scarlatti au Médecin malgré lui de Gounod, en passant par La Flûte enchantée ou par Maria Stuarda de Donizetti, illustrent à point nommé le propos, et les auteurs font toujours preuve d'une érudition jamais pédante. En bons praticiens de la traduction, Apter et Herman reconnaissent d'ailleurs que l'approche, sourcière ou cibliste, privilégiée lorsqu'on commence une traduction, est souvent mise au défi par la pratique: les traducteurs sont obligés de tenir compte de spécificités linguistiques et culturelles (traduction de l'humour, de l'argot, du registre dans lequel les personnages s'expriment, d'ambiguïtés sémantiques...) qui viennent parfois remettre en cause l'optique de traduction initialement choisie.

Enfin, les cinq derniers chapitres (de 8 à 12) sont davantage consacrés aux rapports entre textualité et musique: comment les paroles des personnages, avec leur registre, voire leurs sonorités, s'unissent-elles à la musique pour les caractériser? Ici, les exemples proposés de partitions pour certains personnages wagnériens sont particulièrement passionnants. Que faire, demandent également Apter et Herman, lorsqu'on ne dispose plus de la musique pour certains textes, pour certains chants de troubadours, par exemple, ou lorsque la musique a été détruite ou perdue? Les cha- 
pitres 11 et 12, très fournis, étudient les différentes modifications que la traduction apporte au texte et leur lien à la partition, tout comme le système textuel et musical ainsi créé. Sont alors présentées et largement illustrées les diverses composantes du rythme telles que la syllabe, son accent, sa durée, variables selon les langues, la rime, considérée sous son aspect tant textuel que musical, c'est-àdire employée dans la partition, les répétitions de mots ou de sonorités, et d'autres aspects de l'interaction entre paroles et musique qui prime dans le chant, quel qu'il soit. Enfin, les auteurs rappellent que la traduction doit aussi prendre en compte la tessiture des chanteurs, certains sons se chantant plus aisément dans les aigus, d'autres sur des notes graves. Ils permettent aux lecteurs de bien comprendre à quel point la traduction exige un travail attentif d'écoute, d'empathie avec l'ensemble que constituent le texte et la musique, mais de distance également, afin de mieux en saisir les diverses facettes et leur fonctionnement.

Ils concluent cet ouvrage en souhaitant que la traduction d'opéras vers l'anglais puisse contribuer au développement de ce genre musical... en anglais.

La lecture de ce livre procure beaucoup de plaisir tant il est bien écrit, bien référencé, riche d'exemples et plein d'humour. De plus, il donne envie de réécouter les opéras non seulement dans leur langue originale, mais aussi en traduction, et cela, non seulement vers l'anglais. Il apporte des éléments de réponse à des questions que l'on se posait d'emblée: à quoi sert, à notre époque où de nombreuses salles sont dotées de systèmes de surtitrage, de traduire le chant pour sa performance scénique? Si l'on pouvait penser que la traduction d'opéras était un phénomène dépassé, le livre de Herman et Apter vient remettre en cause ce genre de supposition. L'opéra est certes chanté en version originale en France, mais ce n'est pas toujours le cas ailleurs, et cet ouvrage ouvre une perspective bienvenue sur ce qui se passe dans d'autres pays, rappelant que d'autres traditions sont encore vivaces. Translating for Singing incite à s'interroger sur les valeurs respectives de l'opéra en version originale et en traduction, et sur les apports, plutôt que les «pertes» de cette dernière. Les anglophones ne sont du reste pas les seuls à traduire l'opéra: à Vienne comme à Berlin, deux théâtres majeurs, respectivement la Volksoper et la Komische Oper, présentent encore dans leur programmation pour 2016-2017 plusieurs opéras traduits en allemand. La traduction n'empêche d'ailleurs pas l'emploi du surtitrage (puisque certains de ces spectacles sont présentés traduits en allemand et dotés des surtitres anglais), mais celui-ci est loin de constituer la seule solution.

L’effet général créé par l'interaction entre la musique et le relief sonore des paroles est toujours modifié dans le passage à une autre langue, ce que les auteurs rappellent en fin d'ouvrage. Comme dans toute autre forme de traduction, on aboutit à un produit qui est à la fois même et autre. Ceci n'est pas sans présenter des avantages: écouter les paroles chantées dans plusieurs langues (l'anglais n'est pas la seule langue concernée, loin de là) ouvre non seulement sur une compréhension plus approfondie de l'œuvre, mais encore sur un plaisir auditif à chaque fois nouveau, car différent. En outre, l'appel à la traduction d'opéras vers l'anglais émis par Apter et Herman résonne d'autant plus fort que les œuvres littéraires, elles, sont peu traduites vers l'anglais. Comment cet appel à la traduction d'œuvres chantées s'inscrit-il dans le paysage sociopolitique de la traduction littéraire en général? Que dit-il sur les désirs des divers publics - lecteurs, auditeurs, spectateurs? Et sur le statut de l'anglais comme lingua franca?

Translating for Singing est un bel outil pour quiconque s'intéresse à la traduction du chant (traducteurs, étudiants en masters de traduction, enseignants, chercheurs...), mais il peut s'adresser aussi à toute personne qui étudie la traduction de la poésie ou du théâtre, à la fois par la dimension scénique, orale, articulatoire qu'il met en avant que par celle du rythme et des questions liées à la prosodie (rime, mètres, assonances et allitérations) dont il traite abondamment.

MARIE NADiA KARSKY Université Paris 8 Vincennes Saint-Denis, Saint-Denis, France

GARCIA BARrera, Sebastián (2015): Le traducteur dans son labyrinthe: La traduction de l'Amadis de Gaule par Nicolas Herberay des Essarts (1540). Soria, Facultad de traducción e interpretación, Vertere, 341 p.

García Barrera est maître de conférences en espagnol et en traductologie à l'Université Paris 8. García Barrera est membre de nombreux groupes de recherche dont le Centre d'études et de recherche éditer/interpréter (CÉRÉdl) de l'Université de Rouen, le groupe Cultura, Literatura y Traducción Iber-Artúrica (CLYTIAR) de l'Université de Valladolid en Espagne, et le groupe de recherche en traductologie de l'Université d'Antioquia en Colombie. L'ouvrage est issu de la thèse de doctorat qu'il a rédigée sous la direction du professeur de littérature française Jean-Claude Arnould et défendue à l'Université de Rouen en 2011. Il s'agit d'une analyse historique et descriptive de l'édition française du premier livre de l'Amadis de Gaule traduit de l'espagnol par Nicolas de Herberay, seigneur des Essarts, et paru en 1540. Version 